\title{
Computational simulation of binary compounds of carbon nanotubes and amphiphilics in aqueous solution by Monte Carlo method
}

\author{
L.L. Hermsdorff ${ }^{a}$, C.F.S. Pinheiro ${ }^{a, *}$, A.T. Bernardes ${ }^{a, b}$ \\ a Dep. de Física, Univ. Federal de Ouro Preto, Ouro Preto, MG 35400-000, Brazil \\ b INMETRO, Campus Xerém, Duque de Caxias, RJ 25250-020, Brazil
}

\section{A R T I C L E I N F O}

\section{Article history:}

Received 26 April 2011

Received in revised form 14 February 2012

Accepted 23 February 2012

Available online 29 March 2012

\section{Keywords:}

Monte Carlo methods

Carbon nanotubes

Dissociation

Amphiphilics

\begin{abstract}
A B S T R A C T
Carbon nanotubes have been subject of intensive research because of their outstanding physical properties. The problem of dissolving the aggregation of tubes in bundles formed by strong van der Waals force is addressed in this study. A complete understanding about carbon nanotubes properties is necessary in order to obtain a process of detachment that cause no damage to the tubes. Detachment can be promoted by means of amphiphilic adsorption in water solution. We have studied this phenomenon by means Monte Carlo simulation. The algorithm used was Metropolis algorithm. The analysis of equilibrium was done by means of structure function.
\end{abstract}

(c) 2012 Elsevier B.V. All rights reserved.

\section{Introduction}

Since its discovery in 1991 by lijima [1] carbon nanotubes have been the subject of intense scientific research [2]. This fact occurs due their unique properties such as electrical conduction $\left(\approx 10^{3} \mathrm{~A} /\right.$ $\left.\mathrm{cm}^{2}\right)$, tensile strength $\left(\approx 45 \times 10^{6} \mathrm{~Pa}\right)$, and heat transmission $(\approx 6000 \mathrm{~W} / \mathrm{mK}$, about two times as high as diamond heat transmission) [3-5]. These properties are function of the carbon nanotube morphology, which is defined by diameter and chirality of the nanotube. There is a large number of theoretical and confirmed applications in many areas of science, engineering and other areas [6]. Nevertheless there are some difficulties in reaching this aim. A major challenge is to obtain carbon nanotubes in large quantities at low price by a feasible production method. Another difficulty is the aggregation effect of carbon nanotubes [7]. In most existent methods of production many different kinds of nanotubes are produced. When in solution they are attracted by strong van der Waals forces ( $\approx 500 \mathrm{eV} / \mu \mathrm{m}$ per micrometer of tube-tube contact [8]) forming branches of parallel tubes. The use of carbon nanotubes in a specific application depends on the separation of the nanotubes of the bundle but this is a difficult task to accomplish. An interesting approach is placing the nanotubes in a solution containing surfactants or polymeric structures for wrapping the CNTs and debundling them. Dendritic macromolecules such as PETIM can wrap CNTs and detach them, but a complex is formed altering CNT prop-

\footnotetext{
* Corresponding author. Tel.: +55 31 91272528; fax:+55 3135591667.

E-mail addresses: llhermsdorff@yahoo.com.br (L.L. Hermsdorff), felpin1@gmail. com (C.F.S. Pinheiro), atb@iceb.ufop.br, atbernardes@gmail.com (A.T. Bernardes).
}

erties [9]. Many researchers have tried to promote CNT separation by means of surfactant (or amphiphilic) molecules in aqueous solution $[10,7,8,11-14]$. This process is inexpensive and causes no damage to the nanotube structure that could promote the loss of nanotubes properties. The knowledge about surfactant-carbon nanotube aggregation in aqueous solution process can give important information to resolve the aggregation trouble and allow the use of carbon nanotubes in various applications.

In the present work carbon nanotubes in presence of surfactant molecules in aqueous solution are simulated by Monte Carlo method in different concentrations and temperatures. Our model is bi-dimensional and off-lattice, with Larson-type particles. The algorithm used to attain the equilibrium configuration is the Metropolis Algorithm [15]. The study of the equilibrium has been done by means of the radial distribution function.

\section{Model and simulation details}

All molecules of our model are formed by impenetrable disks with diameter $d$. Each water molecule $(w)$ is represented by a single disk. An amphiphilic molecule is represented by five disks (a head particle $h$ and four tail particles $t$, making a $h_{1} t_{4}$ structure) whose centers are separated by a fixed bond distance equal to $d$. The angle formed by three consecutive bonded particles (or two consecutive bonds) is $109.5^{\circ}$ (regular hexagonal lattice). For simplification only zig-zag helicity $(8,0)$ carbon nanotubes are simulated. The carbon nanotubes $(\mathrm{cn})$ are all considered to have their axes perpendicular to the simulation plane. Therefore the 
nanotubes can be represented by eight disks equidistant from the nanotubes center.

Due its simplicity a water molecule moves only by translation. The amphiphilics move by translation, rotation, reptation and pivoting (see Fig. 1). The carbon nanotubes move by rotation around its own axis and by translation.

Each particle of a molecule can interact with the particles of other molecules. The configurational energy was not considered. The potential between the pairs $h-h, t-t, h-w, t-c n$ and $c n-c n$ are a simplification of the Lennard-Jones potential with a exclusion region of size $d$ and a potential well with a cut distance equal to $3 d$. The potential between the pairs $h-t, t-w, h-n c$ e $w-n c$ has a exclusion region of size $d$ and a repulsive region with size $3 d$. The intensity of each attraction or repulsion for these pairs is $E_{0}$. The pair $w-w$ has a different potential with a exclusion region of size $d$ followed by a repulsion region with size $d$ and energy $6 E_{0}$ and a $-E_{0}$ potential well.

The potentials are represented in Fig. 2. The Hamiltonian of this model is expressed in following equation

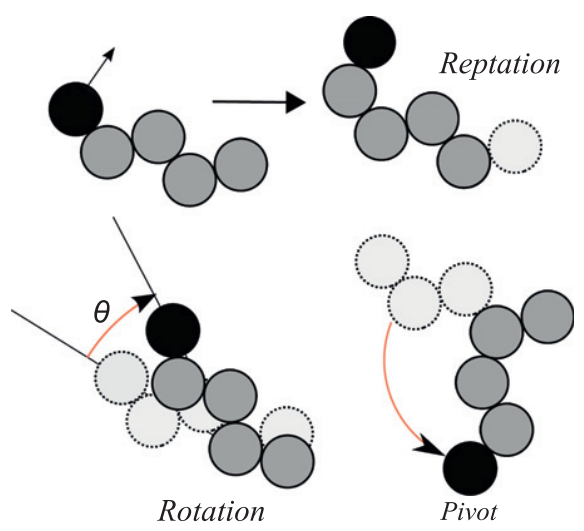

Fig. 1. Amphiphilics allowed movements. In translation, the five disks are displaced by the same vector. In rotation, the center of a randomly chosen disk is the rotation axis. Pivoting happens by choosing a random atom and "bending" the molecule in that site to the other possible angle of $109.5^{\circ}$. In reptation, one of the ends of the molecule moves to a new allowed position so that the others follow it and move to current position of their neighbors in the chain.
Table 1

All possible $S_{i} S_{j}$ products. Repulsion intensities between molecules for which $r_{i j} \leqslant d$ is $\infty$ (hard disk condition).

\begin{tabular}{lllll}
\hline & $w$ & $\mathrm{~h}$ & $\mathrm{t}$ & $\mathrm{nc}$ \\
\hline $\mathrm{w}$ & +1 & +1 & -1 & -1 \\
$\mathrm{~h}$ & +1 & +1 & -1 & -1 \\
$\mathrm{t}$ & -1 & -1 & +1 & +1 \\
$\mathrm{nc}$ & -1 & -1 & +1 & +1 \\
\hline
\end{tabular}

$\mathcal{H}=-\sum_{i, j(i \neq j)} J^{i j}(r) S_{i} S_{j}$

where $r$ is the distance between the centers of two particles $i$ and $j$, $J^{i j}$ is the intensity of the potential between these particles and $S_{i}$ and $S_{j}$ are the $i$ and $j$ particle "charges". The resulting product $S_{i} S_{j}$ is showed in Table 1.

All molecules are inside a square box of size $L$. The boundary conditions adopted is rigid wall. Then, if a molecule will cross the limit of the box the movement is simply rejected.

The movements are performed by first randomly choosing a molecule and then randomly choosing one of the allowed movements to that molecule. Then the new system energy is calculated and the Metropolis condition to the movement is analyzed. A Monte Carlo step (MCS) is defined as the number of tries to move all molecules of the system. The equilibrium is studied by means of the radial distribution function defined by [16-18]:

$g(r)=\frac{\sum_{k=1}^{M} N_{k}(\vec{r}, \Delta \vec{r})}{M\left(\frac{1}{2} N\right) \rho V(\vec{r}, \Delta \vec{r})}$,

where $N_{k}(\vec{r}, \Delta \vec{r})$ is the number of molecules inside a circular layer with internal radius $r$ and external radius $r+\Delta r, M$ is the number of samples, $N$ is the number of molecules in the system and $\rho=\frac{N}{V}$ is the density.

\section{Results and discussions}

The first analysis was performed by simulating water in a box. The box size used in these simulations was $L=40 \mathrm{~d}$. This size was chosen because thermalization is achieved very slowly for low
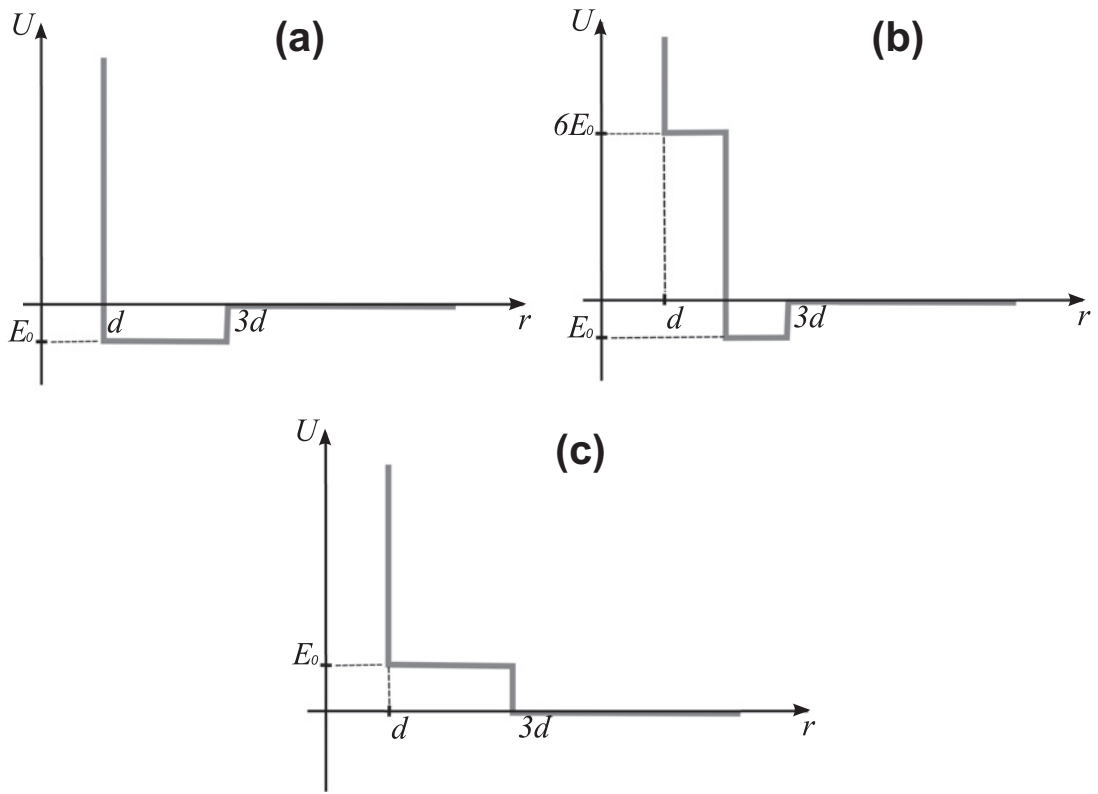

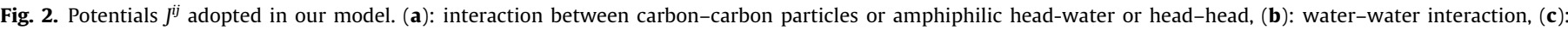
carbon-water interaction. 


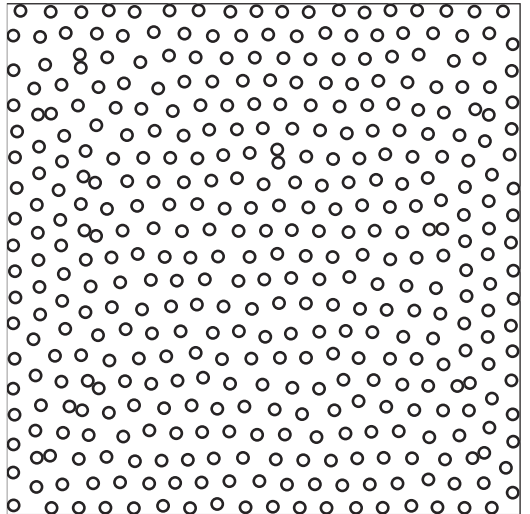

(a)

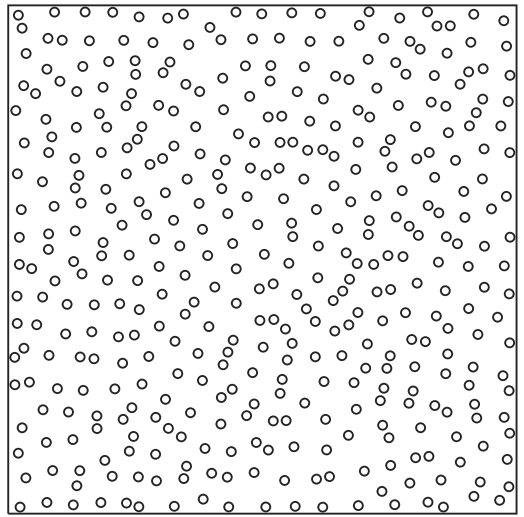

(b)

Fig. 3. Picture of equilibrium of a system with 400 waters: (a) at $T=0.5$ and (b) at $T=1.4$.
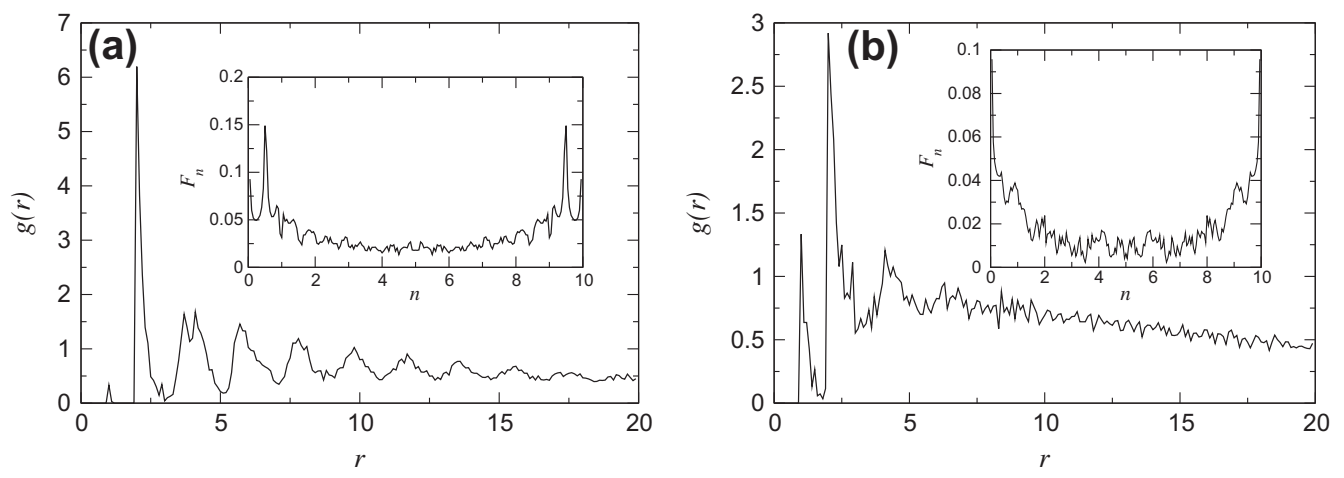

Fig. 4. Results of radial distribution function $g(r)$ and Fourier transform $F_{n}$ (inset) for 400 water molecules after reaching equilibrium: (a) at $T=0.5$ and (b) at $T=1.4$.

temperatures. The temperature utilized in this work is the reduced temperature $T=\frac{k_{b} T_{r}}{E_{0}}$ where $T_{r}$ is the real absolute temperature, $k_{b}$ is the Boltzmann constant and $E_{0}$ is the energy of a water bond. The amount of water in the box was chosen in order to obtain liquidlike behavior. Smaller quantities will make the system behave as a gas. As expected it was found that for lower temperatures the system had an organized structure of a solid phase, and displayed a unordered liquid behavior for higher temperatures. The structure formed when $T<1.0$ is a hexagonal lattice as seen in Fig. 3a for $T=0.5$. In Fig. $3 b$ we see the non-ordered structure of the liquid phase at $T=1.4$. These are pictures of the system we obtained after it had reached equilibrium and they are in agreement with results found in literature $[15,19] .^{1}$

Fig. 4 shows the radial distribution functions for water molecules and their corresponding Fourier transforms for $T=0.5$ (a) and for $T=1.4$ (b). The average separation between molecules is expected to be $2 d$, which is confirmed by the peaks at $r \approx 2 d$ in Fig. 4a and b. Also, the periodicity of peaks in (a), and its absence in (b), confirms the expected distinction between ordered and unordered phases.

A second analysis was made introducing amphiphilic molecules and water in a $L=100 \mathrm{~d}$ box. We performed simulations in different temperatures and amphiphilic concentrations. The concentration definitions used in this work are [20]:

$X_{a m p h}=\frac{N_{a m p h}}{\text { Total volume }(L \times L)}$

\footnotetext{
${ }^{1}$ As for a finite size system it is not possible to see a sharp phase transition, since infinite correlation lengths are not possible.
}

$X_{i}=\frac{\text { Number of aggregates with } i \text { amphiphilics }}{\text { Total volume }(L \times L)}$

$X_{1}^{\text {free }}=\frac{\text { Number free amphiphilics }}{1-3 X_{a m p h}+3 X_{i}}$

Fig. 5 shows $X_{\text {amph }} \times X_{\text {free }}^{1}$ curves for different temperatures. These curves show that there is a critical micelle concentration $(\mathrm{cmc})$. These results are in agreement with literature [21]. Another desired

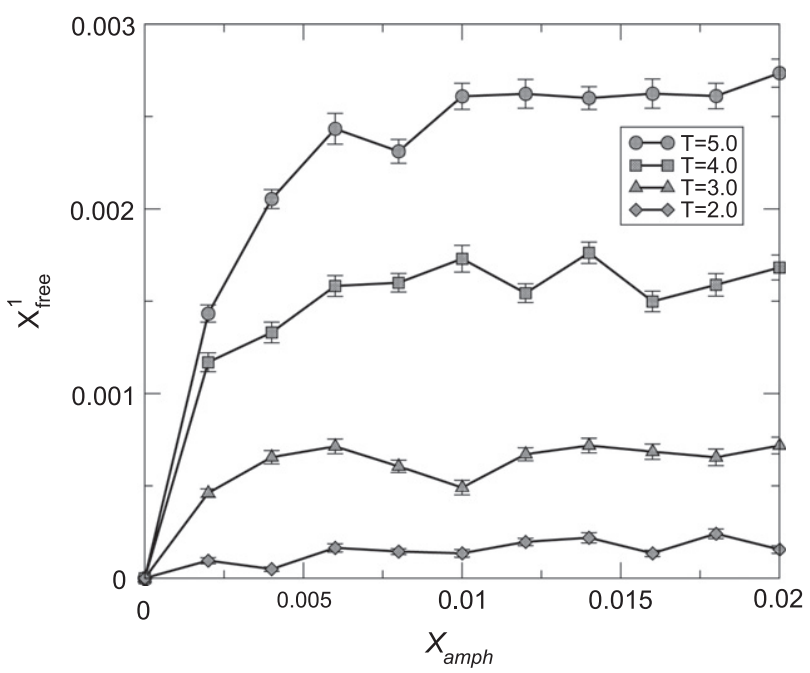

Fig. 5. $X_{a m p h} \times X_{\text {free }}^{1}$ curve for different temperatures. 

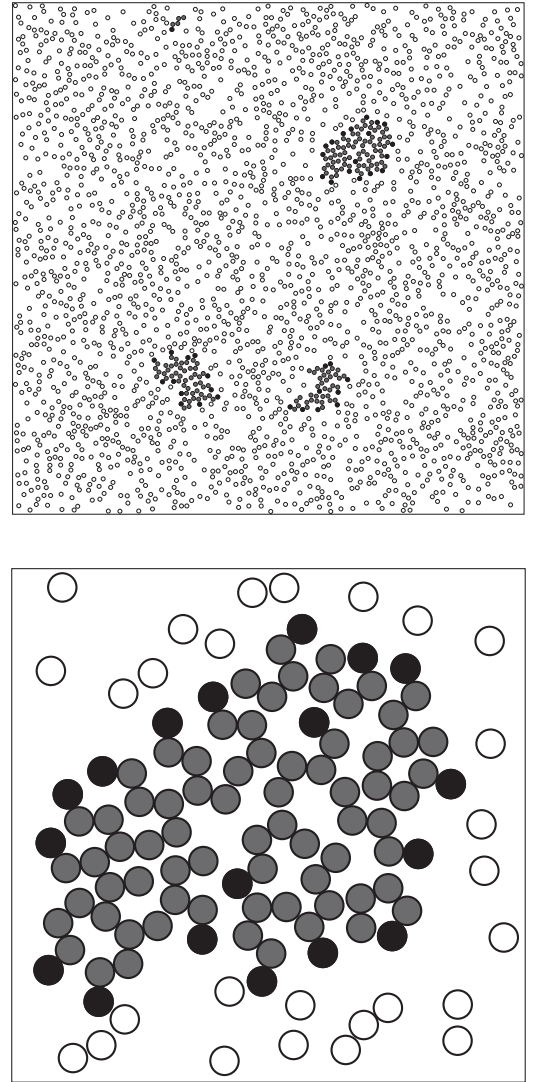

Fig. 6. Top: picture of equilibrium of a system with 2390 water molecules and 40 amphiphilics at temperature $T=3$. Bottom: detail showing a micelle formed by 18 amphiphilic molecules. behavior concerns the aggregation of amphiphilics in typical surfactant equilibrium structures such as micelles, lamellae or vesicles. These structures were observed in our simulations. Micelles appear at small concentrations $\left(X_{a m p h}=0.004\right.$ in temperature $\left.T=0.3\right)$. Lamellae occur at medium concentration (above $X_{a m p h}=0.006$ ). At this concentration micelles are elongated cylinders (cylindrical micelles). Vesicles have appeared only for higher concentrations $\left(X_{a m p h} \geqslant 0.014\right)$. These results show that our amphiphilic model is appropriate, since it agrees with results obtained by other researchers [22]. In Fig. 6 we have a picture of the system after reaching equilibrium, with 2380 water molecules and 40 amphiphilics at temperature $T=3$, and the details around a micelle.

The simulations with nanotubes were carried out in a $L=100 \mathrm{~d}$ box in presence of water and different concentrations of amphiphilics. Simulations with one, two and three nanotubes were performed. In simulations with 1 nanotube the aggregation of nanotubes cannot take place. Nevertheless this situation is appropriate to study the amphiphilic-nanotube aggregate in thermal equilibrium. In small concentrations and temperature $T=3.0$ the amphiphilics form aggregates like micelles around the nanotube. An interesting phenomenon is that elongated micelles here occur at concentrations lower than before, when nanotubes were absent. This suggests that nanotubes make lamellae and vesicle formation more likely. At higher concentrations, the amphiphilics form deformed lamellae aggregates around the nanotubes. For higher concentrations the aggregates formed by amphiphilics are deformed vesicles. Fig. 7 shows some of these structures. Fig. 8 shows the results of radial distribution function for different concentrations. In these curves $r$ is the distance between an amphiphilic head and the nanotube center. Note that the curve of $X_{a m p h}=0.006$ shows a high density of amphiphilics at farther distances from nanotubes. This reveals the presence of a lamella around the carbon nanotube. The $X_{\text {amph }}=0.002$ curve has a maximum around $r=6,5 d$. This dis-
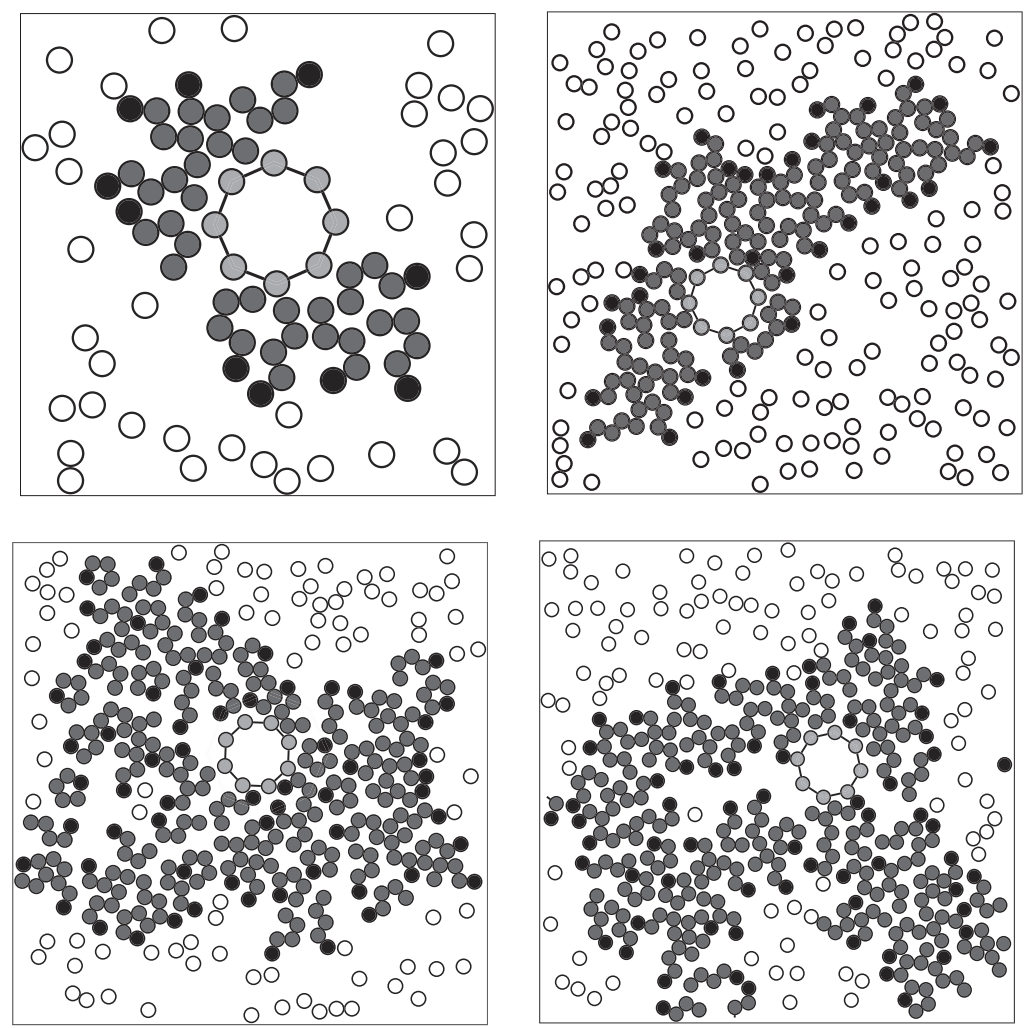

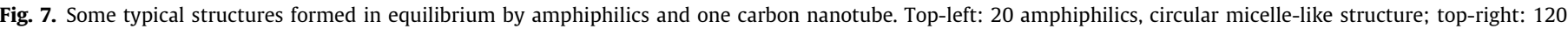
amphiphilics, elongated micelle structure; left-bottom: 160 amphiphilics, vesicle-like structure; right-bottom: 200 amphiphilics, vesicle-like structure. 
tance is approximately the sum of the amphiphilic size and the nanotube radius $\left(r_{n c}+d_{a m p h} \simeq 6.3 d\right)$. It shows that the amphiphilics stand strained with the tail end near the nanotubes surface for small concentrations. In higher concentrations the maximum occurs at smaller $r$ values. For these concentrations the aggregate around the nanotube is larger. This explains why the formation of a strained configuration of amphiphilics is more difficult.

Fig. 9 shows the results of the radial distribution function averaged over 40 samples obtained after thermal equilibrium. These images show some peaks around $r=2.5$ meaning the amphiphilic head is near the nanotube surface. According to the hamiltonian describing the system, that is an unfavorable energetic situation. This result suggests that the nanotube deforms the amphiphilic aggregate when it penetrates the aggregate. These amphiphilics stand in this situation and cannot easily change their configuration. Fig. 10 shows pictures of the thermal equilibrium with different kinds of aggregates. At $T=4.0$ the system behaves similarly to $T=3.0$ only for higher amphiphilics concentrations. At temperature $T=5.0$ the amphiphilics do not form aggregates around the nanotubes.

The simulations with two nanotubes always started with nanotubes placed touching each other. In aqueous solution without amphiphilics the nanotubes do not dissociate. Dissociation process will only take place when the nanotubes do not interact with each other, which occurs for distances greater than $3 d$. Then two nanotubes are dissociated when the minor distance between a particle of one nanotube and a particle of the other nanotube is greater than $3 d$. At temperature $T=3.0$ dissociation does not occur for
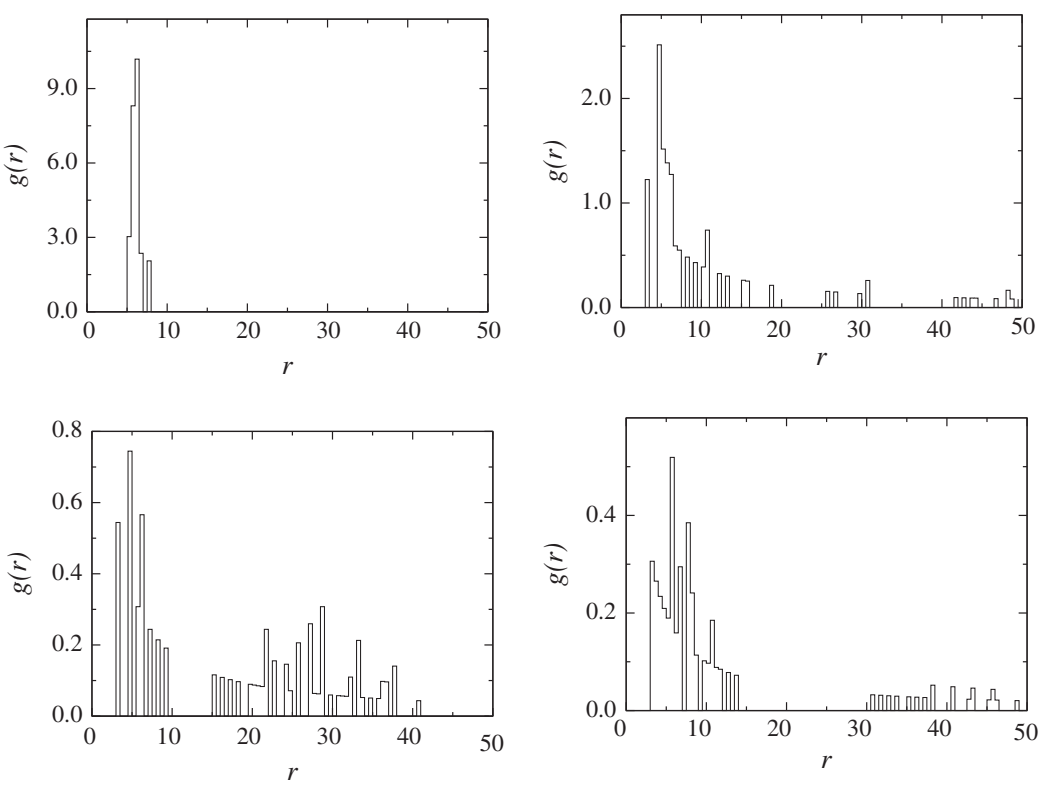

Fig. 8. Radial distribution function for systems with 20,40,60 and 80 amphiphilics.
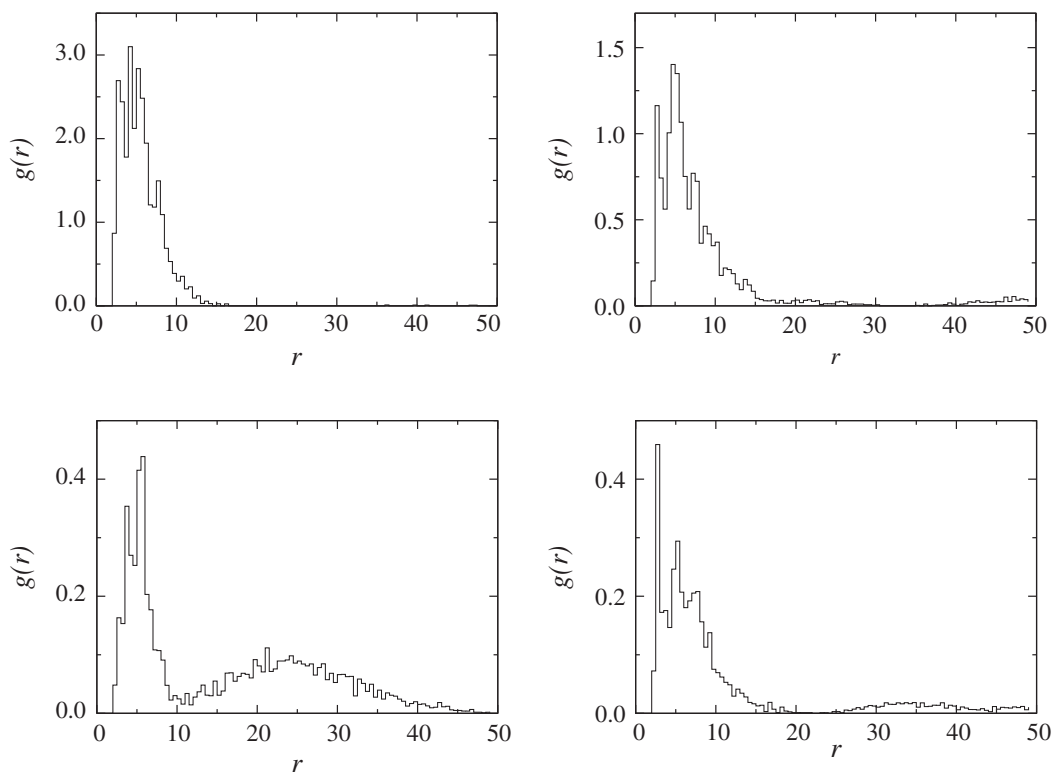

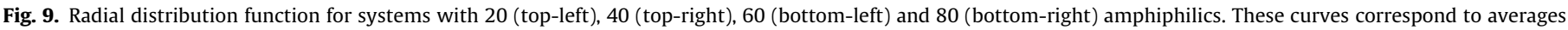
over 40 samples. 

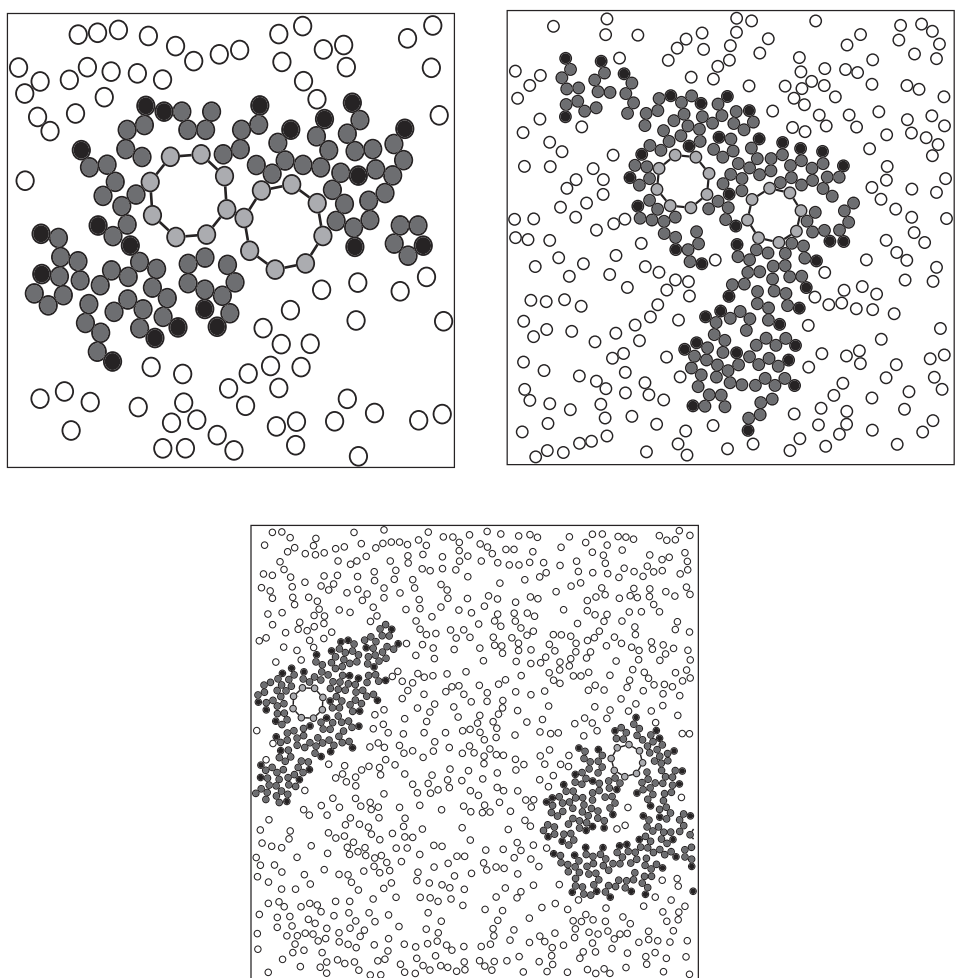

Fig. 10. Structures formed in equilibrium. Left: 60 amphiphilics, right: 100 amphiphilics, bottom: 180 amphiphilics.

small concentrations. In our simulations with $X_{a m p h}=0.006$, there is enough amphiphilic to form an aggregate that could cause separation, yet the aggregates that are formed are not bulky enough. Only for concentrations above $X_{a m p h}=0.008$ dissociation occurs. In all simulation with $X_{a m p h} \geqslant 0.008$ carbon nanotube dissociation was observed. In higher amphiphilics concentrations vesicles appear in the system and the nanotubes stay inside the vesicles.

In simulations with 3 nanotubes the same concentration thresholds were observed for dissociation. At concentrations below $X_{a m p h}=0.008$ the nanotubes stay together. This implies the determinant factor for this dissociation is the capacity of the amphiphilics to form big enough aggregates. In other words this capacity is determined by the number or fraction of amphiphilics in the system. This result has already been reported in a recent experimental work [23].

\section{Conclusions}

The separation of carbon nanotubes from the bundles of tubes by use of amphiphilic molecules in aqueous solution was studied with Monte Carlo Simulation. Our model actually showed some characteristics of this detachment. Detachment only took place for larger amphiphilic concentrations. The determinant characteristic that defines the detachment in our model is the capacity of the formation of big aggregates of amphiphilic molecules around the nanotubes. The amphiphilics surrounding the nanotubes are responsible for this detachment. This detachment process is suitable given its simplicity concerning materials, not to mention the low costs involved. Nevertheless, this process does not destroy or modify the nanotubes structure, keeping their properties intact. With our model we found that the concentration of amphiphilics in the system defines how effective is the detachment of carbon nanotubes, which is corroborated by recent experimental results [23].

\section{Acknowledgments}

The authors are thankful to the national agencies Fapemig, $\mathrm{CNPq}$ and Capes for financial support.

\section{References}

[1] S. Iijima, Nature 354 (1991) 56-58, doi:10.1038/354056a0.

[2] G. Mountrichas, N. Tagmatarchis, S. Pispas, The Journal of Physical Chemistry B 111 (29) (2007) 8369-8372, doi:10.1021/jp067500k (pMID: 1738845)

[3] D. Tomanek, Preface and introduction, in: A. Jorio, M. Dresselhaus, G. Dresselhaus (Eds.), Carbon Nanotubes: Advanced Topics in the Synthesis, Structure, Properties and Applications, Topics in Applied Physics, vol. 111, Springer, Berlin, 2008

[4] P.G. Collins, P. Avouris, Scientific American 283 (2000) 62-69.

[5] R.H. Baughman, A.A. Zakhidov, W.A. de Heer, Science 297 (5582) (2002) 787792, doi:10.1126/science.1060928.

[6] J. Chen, M.A. Hamon, H. Hu, Y. Chen, A.M. Rao, P.C. Eklund, R.C. Haddon, Science 282 (5386) (1998) 95-98, doi:10.1126/science.282.5386.95. arXiv:http:// www.sciencemag.org/content/282/5386/95.full.pdf, URL <http:// www.sciencemag.org/content/282/5386/95.abstract>.

[7] V.A. Sinani, M.K. Gheith, A.A. Yaroslavov, A.A. Rakhnyanskaya, K. Sun, A.A. Mamedov, J.P. Wicksted, N.A. Kotov, Journal of the American Chemical Society 127 (10) (2005) 3463-3472, doi:10.1021/ja045670.

[8] M.J. O’Connell, S.M. Bachilo, C.B. Huffman, V.C. Moore, M.S. Strano, E.H. Haroz, K.L. Rialon, P.J. Boul, W.H. Noon, C. Kittrell, J. Ma, R.H. Hauge, R.B. Weisman, R.E. Smalley, Science 297 (5581) (2002) 593-596, doi:10.1126/science.1072631.

[9] G. Jayamurugan, K.S. Vasu, Y.B.R.D. Rajesh, S. Kumar, V. Vasumathi, P.K. Maiti, A.K. Sood, N. Jayaraman, The Journal of Chemical Physics 134 (10) (2011) 104507, doi:10.1063/1.3561308. URL <http://link.aip.org/link/?JCP/134/ $104507 / 1>$.

[10] B. Koh, J.B. Park, X. Hou, W. Cheng, The Journal of Physical Chemistry B 115 (11) (2011) 2627-2633, doi:10.1021/jp110376h. arXiv:http://pubs.acs.org/ doi/pdf/10.1021/jp110376, URl <http://pubs.acs.org/doi/abs/10.1021/ jp110376h>

[11] R. Duggal, M. Pasquali, Physical Review Letters 96 (24) (2006) 246104, doi:10.1103/PhysRevLett.96.246104.

[12] Y. Kang, T.A. Taton, Journal of the American Chemical Society 125 (19) (2003) 5650-5651, doi:10.1021/ja034082d (pMID: 1273390).

[13] R. Bandyopadhyaya, E. Nativ-Roth, O. Regev, R. Yerushalmi-Rozen, Nano Letters 2 (1) (2002) 25-28, doi:10.1021/nl010065f.

[14] S. Bhattacharyya, J.-P. Salvetat, D. Roy, V. Heresanu, P. Launois, M.-L. Saboungi, Chemical Communications (2007) 4248-4250, doi:10.1039/B709499J. URIL <http://dx.doi.org/10.1039/B709499J>. 
[15] E. Teller, N. Metropolis, A. Rosenbluth, Journal of Chemical Physics 21 (13) (1953) 1087-1092.

[16] N. Metropolis, A.W. Rosenbluth, M.N. Rosenbluth, A.H. Teller, E. Teller, Journal of Chemical Physics 21 (1953) 1087-1092.

[17] A.T. Bernardes, T.B. Liverpool, D. Stauffer, Physical Review E 54 (3) (1996) R2220-R2223, doi:10.1103/PhysRevE.54.R2220.

[18] T. Megyes, S. Bálint, I. Bakó, T. Grósz, T. Radnai, G. Pálinkás, Chemical Physics 327 (2-3) (2006) 415-426, doi:10.1016/j.chemphys.2006.05.014.

[19] A. Lehninger, D. Nelson, M. Cox, et al., Principles of Biochemistry, Worth Publishers, New York, 1982.
[20] A.T. Bernardes, V.B. Henriques, P.M. Bisch, The Journal of Chemical Physics 101 (1) (1994) 645-650, doi:10.1063/1.468120.

[21] S. Yang, X. Zhang, S. Yuan, Journal of Molecular Modeling 14 (2008) 607-620, doi:10.1007/s00894-008-0319-7. URL <http://dx.doi.org/10.1007/s00894008-0319-7>.

[22] E.D. Goddard, G.C. Benson, Transactions of the Faraday Society 52 (1956) 409413, doi:10.1039/TF9565200409.

[23] A.J. Blanch, C.E. Lenehan, J.S. Quinton, The Journal of Physical Chemistry B 114 (30) (2010) 9805-9811, doi:10.1021/jp104113d. 